Gynaecologia 1957;144(Suppl. 1):I-VI

\title{
Contents, Vol. 144, Supplement 1, 1957
}

\section{Liste des communications}

Gynécologie generate

Funck-Brentano, P. (Paris): L'endométriome du colon sigmoide

Discussion: ,/. de, Brux, R. Bourg, P. Funck-Brentano

Bédrine, H., et Houcke (Lille): A propos d'un neurinome pelvien

Keller, R., et B. Keller (Strasbourg): Quelques reflexions au snjet du traitement du prolapsus genital

survenu après hystérectomie

Discussion: E. Ghomé, R. Palmer, H. Robert, M. Magendie, R. Bourgde Brux, J. (Paris):

Thromboses fibrineuses et physio-pathologie génitale

Discussion: J. BretBret, A. ./. (Paris): Métrorragies et phlébites pelviennes

Discussion: J. Bourg, R. Gilbert-Dreyfus, J. de Brux, II. Pigeaud

Stamm, H. (Bale): Prophylaxie postopératoire au moyen de la Butazolidine

Sigg, K. (Bale): Traitement des thromboses superficielles et profondes et $\Gamma$ application de

Butazolidine

Netter, A., H. Yaneva et A. Lambert (Paris): Les ménocytes

Discussion: J. de, Brux, A. Netter Magendie, J., J. Ma-squ, elier et P. Nocquet (Bordeaux):

Enquête sur la valeur de la technique des anti-

biogrammcs en gynécologie

Discussion: R. Palmar, M. Magendie, J. Bret, J. MasquelierMagendie, ./., J. Yincendeau et M.

Régnier (Bordeaux): Gynécomastie et tumeur du testicule

Discussion: J. @ilbert-DreyfusPulsford, J. (Paris): Hystérosalpingographie et enregistrement manométrique

Discussion: F. PapiUon, J. Pulsford, R. PalmerFerin, J. (Louvain): Les efl $1 / 8$ ts ehez la femme des esters de 19-nortostostérone

Discussion: R. Vokaer, M. de Graef, J. Ferin, R. Bourg, A. Netter, R. Wenner

Cervicit,e et vaginite

PapiUon, F. A. (Paris): Dix années de pratique et de recherche systématique du pH vaginal au cours de $\Gamma$ examen gynécologique

Pundel, J. P., et F. Schwacht, gen (Luxembourg): Le terrain hormonal des infections vaginales à tricho-

monas

Discussion: J. Dalsace, J. P. Pundel, J. de Brux

Mutter, P. (Strasbourg): Reflexions au sujet de quelques cas de cervicites au cours de la grossesse ....

Cancer utérin

Moricard, R., S. Өothié et J. Belaisch (Paris): T)e I'utilisation des traoeurs et en partioulier du 35 $\mathrm{S}$

comme moyen d'étude de la secretion cervicale 
Discussion: J. de Brux, Mile Gothic

$\mathrm{V}$

de Brux, J. (Paris): Les vestiges embryonnaires du traotus genital féminin. Leur role dans la stérilité

et la formation des grossesses extra-utérines

55

Pundel, J. P., et C. Lichtfus (Luxembourg): Modifications de la coloration cytologique des frottis vaginaux à $\Gamma$ hématoxyline-Shorr 58

Berger, J., et L. 0. Zwillenberg (Bale): Observations sur le stratum cylindricum de Гépithélium pavimen-

teux du col utérin à Гaide d'une coloration «argent-polychrome»

Ginglinger, A., et R. Gandar (Strasbourg): Difficultés de diagnostic et traitement de

Гépithólioma intra-

épithólial du col utérin

70

Discussion: F. A. Papillon, R. Palmer, E. Douay, J. de Brux Palmer, R. (Paris): La biopsie

exérèse à l'anse diathermique des lesions bénignes et suspectes du col utérin

(résultats-technique) 72

Discussion: J. Magendie Laffont, A., et M. Bonafos (Alger): Les hormones dans le traitement des complications post radiothéra-

piques des cancers utérins

74

Birth-control, discussion

Merz, W. R.: Problèmes de Гavortement thérapeutique et de Tanticonception

Discussion: E. Douay, A. Pecker, R. Pigeaud, Th. Roller, J. Dalsace, E. Ghomé, P. Vellay

Berger, J., et M. Mall (Bale): A propos de la grossesse involontaire

83

Discussion: Th. Roller

Obstétrique

Caderas de Rerleau, J. (Montpellier): Conduite à tenir au cas d'iso-immunisation par le facteur rhesus . . 87

Mayer, M., et P. Ducas (Paris): Traitement préventif des accidents d'iso-immunisation anti-Rh par

Thormonothérapie surrénalienne

Discussion: A. Hotting er

Javelier, A. (Paris): Emploi du Largactil dans le traitement des vomissements gravidiques 93

Lévy-Solal, E., et P. Morin (Paris): Des anomalies fonctionnelles de Гutérus parturient contrôlécs par

la vecto-utérographie 96

Discussion: P. Burger, P. Morin Lepage, F., et 8. Verte (Paris): Les anomalies transitionnelles de la charnière lombo-sacróe element de

pronostic de $\Gamma$ épreuve du travail dans certains bassins limites

Ghomé, E., et J. Ghomé (Paris): Quelques considerations sur la physiologic motrice du col de Гutérus. . 104 Gosselin, 0. (Liege): La ponction abdominale de Гæuf au cours de $\Gamma$ accouchement dans les grossesses

hydramniotiques

Burger, P. (Strasbourg): La rupture uterine du placenta increta Ill

de Rom, F. M. F. (Gand): La mortalité infantile périnatale (M.I.P.) 113 
Broquet, G. (Bale): A propos de quatre observations ayant prósenté un «Supine Hypotensive Syndrome » 116

Gynecologic psychosornatique

Michel-Wolfromm, H. (Paris): Algies pelvienncs psychogènes 119

Hauser, A. (Bale): Système nerveux végétatif et algies pelviennes 121

Accouchement psychosornatique

$\dagger$ Lamaze, F., et P. Vellay (Paris): Psycho-prophylaxie et travail dirigé 125

Discussion: A. Laffont, P. Rochat, P. Vellay, R. Bourg, P. A. Papillon Pigeaud, H. (Lyon):

Accouchement naturel et preparation psycho-physiothérapique prophylactique . 129

Discussion: P. Vellay, P. Burger Merz, W. R. (Bale): Influence de la gymnastique et de la psychoprophylaxie sur dift'órents facteurs ob-

joctivs do Taccouchement 132

VI

Tiiberculo.se génitale

Roller, Th. (Bale): Quelques considerations sur la tuberculose génitale de la femme

Dalsace, J. (Paris): Existe-t-il un aspect radiologique pathognomoiiique de la tuberculose génitale in-

apparente ? 137

Gosselin, 0., Beginster et Ghandelle (Liège): A propos du diagnostic de la tuberculose génitale 139

Discussion: E. Chomé, 0. Gosselin, A. Netter, J. de Briix, B. Гapillon. J. Bret, J. Dalsace

Diagnostic et thérapie hormonales

Aschheim, 8. (Paris): Sur la stabilité des hormones gonadotropes dans leurs tissus d'origine 142

Wenner, B., G. A, Hauser et M. Keller (Bale): Dysgénésie des gonades 14õ

Discussion: J. Gilbert-Dreyfus, A. Netter Gilbert-Dreyfus, J., J. G. Savoie, J. Sebaoun et J. Ph. Mery (Paris): Un nouveau type d'exploration dyna-

mique surréno-ovarienne: valeur et signification dans un eas de virilisme par arrhénoblastome .... 146

Discussion: M. JayleJayle, M.-F., et ./. Gueguen (Paris): Nouveau mode d'exploration dynamique du corps jaune 148

Discussion: S. Aschheim, B. Bourg, M. JayleBernard, I., et Mile Neuville (Bordeaux): Le rhumatisme endocrinien 153

Discussion: J. Bret, M. Jayle.Bret, A.-J. (Paris): Les avortements avec hyperandrogénie.

Traitement par la cortisone 158

Discussion: M. Jayle, A. Netter Schoysnia.n, B. (Bruxelles): Etude expérimentale de Гactivité séorétoire de la vésicule séminale du rat.

Note préliminaire $\quad 161$

Da Bugna, D. (Bale): Quelques reflexions sur la stérilité masculine 163

Bret, A.-J. (Paris): A propos de Thyperandrogénie. Troubles menstruels et cortisone

Demonstrations Koller, Th. (Bale): Kyste géant se développant à partir des vestiges

embryonnaires du canal de Wolff. 168 Preface

C'est du 2 au 4 juillet 1956 qu'eurent lieu, à la Clinique Universitaire de Gynécologie et d'Obstétrique de Bale, les Journées Franco-Belgo-Suisses. Ce congrès, auquel furent invites un certain nombre de gynécologues et d'accoucheurs français et beiges éminents, avait pour but de reserrer, dans le cadre d'une même spécialité, les liens tant scienti-fiques que culturels, aussi 
bien par des conferences ou des discussions que par des contacts personnels et amicaux. Les rapports et discussions, groupés par themes, paraissent dans le present numéro special de «Gynaecologia», que nous nous faisons un plaisir de faire parvenir à titre gracieux à tous les participants étrangers.

Au sommaire, nous voyons que quelques chapitres très particuliers furent discutés. Mentionnons entre autres le cancer de I'uterus, la discussion au sujet du birth - control, la gynécologie $\varphi$ sycho-somatique, Vaccouchement sans douleur (en partie avec films), la tuber-culose génitale, ainsi que quelques chapitres d'hormonologie.

Les organisateurs des «Journées» Th. Roller, R. Wenner, W. R. Merz, J. Berger. P. Puisse ce numéro special nous rappeler longtemps encore ces rencontres amicales, carac-térisées non seulement par une partie scientifique intéressante, mais 